\title{
CALORIMETRIC ABSORPTION SPECTROSCOPY OF DEEP DEFECTS AND QUANTUM DOTS
}

\author{
R. Heitz, L. Podlowski, J. Böhrer, A. Hoffmann, I. Broser \\ AND D. BIMBERG
}

Technische Universität Berlin, Institut für Festkörperphysik, 10623 Berlin, Germany

In recent years calorimetric absorption spectroscopy has been developed to a powerful tool of semiconductor spectroscopy based on the detection of nonradiative relaxation processes. Calorimetric absorption spectroscopy is an ultrasensitive quantitative absorption technique. Recent investigations of $\mathrm{Fe}$ in III-V semiconductors and of InAs/GaAs quantum dots are presented here to illustrate the potential of the method. Sharp absorption lines are observed at the low energy onset of the $\mathrm{Fe}^{3+/ 2+}$ charge transfer band in III-V semiconductors. Calorimetric absorption spectroscopy measurements in the $\mathrm{mK}$ range reveal a strong temperature dependence of their absorption strength identifying unambiguously $\mathrm{Fe}^{3+}\left({ }^{6} A_{1}(S)\right)$ as a ground state. The excited state is attributed to $\left(\mathrm{Fe}^{2+}, \mathrm{h}\right)$. The importance of exchange interaction for the observed fine structure is pointed out and binding energies are determined. The quantum yield of the intracenter ${ }^{5} T_{2}-5$ transition of $\mathrm{Fe}^{2+}$ is determined to be below $50 \%$ at $2 \mathrm{~K}$. A correlation between the nonradiative relaxation rate and the isotope splitting of the ${ }^{5} T_{2}-{ }^{5} E$ transition is observed, demonstrating the crucial role of the dynamical Jahn-Teller coupling of the ${ }^{5} T_{2}$ state to local $T_{2}$ modes for the multiphonon relaxation process. Quantum dots having a $d$-function density of states should exhibit no Stokes shift between absorption and emission as observed for one- and two-dimensional systems. Calorimetric absorption spectroscopy demonstrates ground state absorption coinciding in energy with the luminescence for self- organized InAs/GaAs quantum dot structures grown by MBE. Transitions into excited hole states are resolved and a comparison to photoluminescence excitation spectroscopy is presented.

PACS numbers: $71.55 . \mathrm{Eq}, 71.70 . \mathrm{Gm}, 73.20 . \mathrm{Dx}$

\section{Introduction}

Phonons play a major role for the relaxation of excited semiconductors. Despite their omnipresence only few experimental techniques are based on their detection $[1,2]$. In the last decade calorimetric absorption spectroscopy (CAS) [2-4] has been developed to a highly sensitive absorption technique which is superior 
to other photothermal techniques, e.g. photoacoustic spectroscopy and photothermal deflection spectroscopy. A detailed comparison of different photothermal techniques has been given by Bimberg et al. [3] recently. CAS being the most sensitive one oflers the additional advantage of being quantitative. E.g., CAS allows one to quantify the power dissipated in the sample by nonradiative processes giving access to quantum efficiencies or to determine absolute absorption coefficients. CAS was introduced in 1981 [2] and subsequently continuously improved its sensitivity in particular by reducing the basis temperature to the $\mathrm{mK}$ range $[3,4]$. In recent years CAS has been successfully employed to investigate absorption processes of deep centers like transition metals in II-VI and III-V semiconductors [5, 6] and to study absorption in III-V nanostructures including type-II systems $[7,8]$.

In this contribution we first describe the basics of CAS. In the main part CAS results on Fe doped III-V bulk semiconductors are discussed giving a detailed insight into the properties of charge transfer states of the Fe center and nonradiative relaxation processes of $\mathrm{Fe}^{2+}$. In the last part we demonstrate the potential of CAS to detect weak absorption of a single InAs/GaAs quantum dot (QD) layer.

\section{Calorimetric absorption spectroscopy}

CAS measures the increase in sample temperature upon optical excitation. All phonons generated in nonradiative relaxation processes are integrally detected. The sensitivity of CAS is usually sufficient to employ low power optical light sources, e.g. a tungsten halogen lamp in connection with a high resolution monochromator, having the advantage of a wide tuning range. The most important part of a CAS setup is the sample/thermometer arrangement depicted schematically in Fig. 1. The sample is mounted thermally isolated in a Ile cryostat with nylon threads of low thermal conductivity. A Cu wire establishes a good thermal link between the sample and the sapphire chip accommodating the low temperature thermometer and a Au resistor. A weak thermal link of resistance $R_{\mathrm{L}}$, e.g. realized

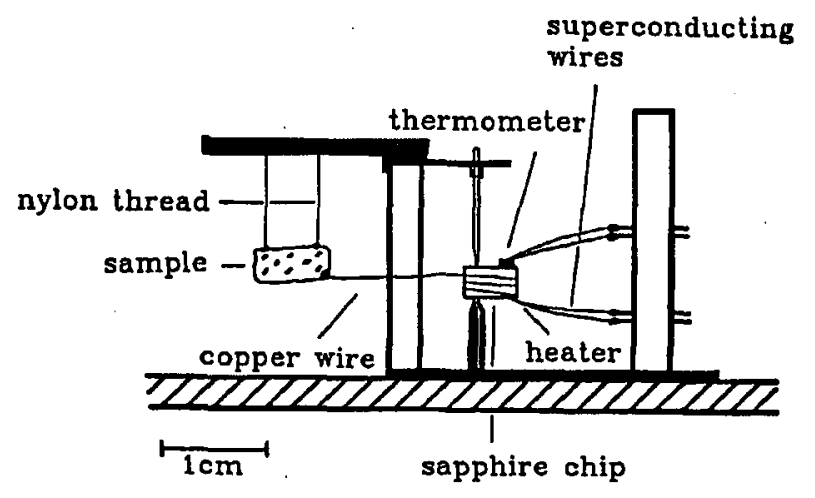

Fig. 1. Sample/thermometer arrangement for calorimetric absorption spectroscopy. Similar setups with a black body as an absorber are used to mcasure transmission or reflection spectra. 
by a $\mathrm{Cu}$ tip (Fig. 1) or a manganin wire, leads to a homogeneous temperature in the sample/thermometer arrangement of heat capacity $C$. The Au resistor serves for calibration of the calorimeter by controlled heating and, thus, the CAS signal is not given as temperature rise but as equivalent heating power $P_{\mathrm{CAS}}$. The temperature rise $\Delta T$ and the corresponding time constant $t$ of the sample/thermometer arrangement are given by

$$
\Delta T=\Delta P_{\mathrm{CAS}} R_{\mathrm{L}} \quad \text { and } \quad \tau=R_{\mathrm{L}} C .
$$

The upper limit of $\tau$ equals a few seconds given by the need to measure spectra. In order to optimize $\Delta T$ the basis temperature of the setup has to be reduced. The heat capacity $C$ decreases like $T^{3}$ allowing for a higher thermal resistance $R_{\mathrm{L}}$ increasing $\Delta T$. In practice the resistance change of the low temperature thermometer is measured, which is linked to $\Delta T$ by the sensitivity $G$

$$
\frac{\Delta R}{R}=G \Delta T \text {. }
$$

$G$ increases with decreasing temperature [3]. In recent years, subsequently CAS setups in ${ }^{4} \mathrm{He}[2],{ }^{3} \mathrm{IIe}[3]$ and ${ }^{3} \mathrm{He} /{ }^{4} \mathrm{He}$ dilution [4] cryostats operating at $1.3 \mathrm{~K}$ (carbon resistor), $500 \mathrm{mK}$ (Ge resistor) and $45 \mathrm{mK}\left(\mathrm{RuO}_{2}\right.$ resistor), respectively, have been developed. Therewith the detection limit has been improved from $250 \mathrm{pW}$ to $100 \mathrm{rW}$. CAS detects that part of the exiting light power $P_{\text {in }}$ absorbed in the sample and transferred to the phonon system yielding the external quantum efficiency $Q$

$$
Q=1-\frac{P_{\mathrm{CAS}}}{P_{\mathrm{abs}}} \quad \text { and } \quad P_{\mathrm{abs}}=P_{\mathrm{in}} \frac{(1-R)\left(1-\mathrm{e}^{-\alpha d}\right)}{1-R \mathrm{e}^{-\alpha d}},
$$

with $\alpha d$ the optical thickness of the sample and $R$ its reflectivity. As long as $Q$ remains constant $P_{\mathrm{CAS}}$ increases with $\alpha d$ and, thus, CAS can be employed as a highly sensitive absorption technique. Comparing absolute values the external quantum efficiency $Q$ of the sample can be determined.

\section{Fine structure of the iron charge transfer band}

Fe-doped III-V semiconductors exhibit broad absorption bands associated with charge-transfer processes of the Fe center [9]. Transitions involving the deep $\mathrm{Fe}^{3+/ 2+}$ acceptor level dominate the spectra. The first observation of sharp lines at the low energy onset of the $\mathrm{Fe}^{3+/ 2+}$ photoionization bands in semi-insulating InP:Fe [5] has triggered a lot of further investigations, e.g. [10-12]. Nevertheless, only a qualitative understanding of the fine structure states has been achieved yet.

Figure 2 depicts CAS spectra of semi-insulating InP and GaP doped with Fc. Fine structure resolved at the low energy onset of the $\mathrm{Fe}^{3+/ 2+}$ charge transfer band is shown on an enlarged energy scale in the insets. The most pronounced absorption structure is a quintet labeled $a^{\prime}-e^{\prime}$ around 771 and $825 \mathrm{meV}$ in InP and GaP, respectively. On the high energy side the broad band labeled $T A$ is attributed to an acoustical phonon sideband of $a^{\prime}-e^{\prime}$. The origin of the doublet labeled $a, b$ in both $\operatorname{InP}$ and $\mathrm{GaP}$ is controversial. The doublet has been attributed to further electronic transitions [12] or to phonon replica of the quintet $a^{\prime}-e^{\prime}[10]$.

Temperature dependent investigations in the $\mathrm{mK}$ range yield information on the origin of the fine structure shown in Fig. 2. No changes of the fine structure 


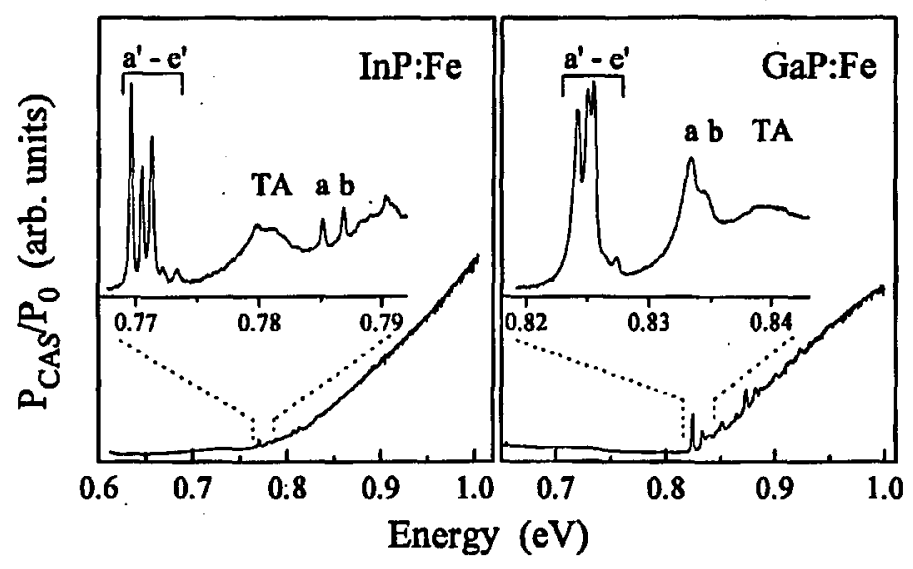

Fig. 2. CAS spectra of semi-insulating $\mathrm{InP}$ and $\mathrm{GaP}$ doped with Fe. Insets give the fine structure occurring at the low energy onset of the $\mathrm{Fe}^{3+/ 2+}$ photoionization band on an enlarged energy scale.

spectra can be observed between 0.5 and $10 \mathrm{~K}$. The resolved splittings reflect the fine structure of the final state of the transitions. This situation, however, is different if the temperature is decreased to the $100 \mathrm{mK}$ range. The left part of Fig. 3 compares high resolution spectra for GaP:Fe recorded at basis temperatures between 45 and $160 \mathrm{mK}$. The decreasing signal to noise ratio with increasing temperature reflects the decreasing sensitivity of the setup. The relative intensities within the quintet $a^{\prime}-e^{\prime}$ and of the doublet $a, b$ vary with temperature, whereby the $160 \mathrm{mK}$ spectrum resembles a typical "high" temperature spectrum. The right part of Fig. 3 gives the development of the integral intensity of the fine structure lines being proportional to the absorption coefficient obtained from line shape fits.

The change of the absorption coefficient at $\mathrm{mK}$ temperatures results from thermalization in the ground state $(E)$. The thermal energy $k T$ is about $10 \mu \mathrm{eV}$ for the investigated temperature range, which is much smaller than the linewidth of the optical transitions. Each absorption line is the superposition of two transitions having different states as indicated in the inset of Fig. 3. Both transitions contribute to $P_{\mathrm{CAS}}$ corresponding to the equilibrium ground state population and the respective oscillator strength $f_{i}$

$$
I_{i}=\frac{A f_{1 i}}{1-\frac{g_{2}}{g_{1}} \mathrm{e}^{-\Delta E / k T}}\left\{\frac{f_{2 i}}{f_{1 i}} \frac{g_{2}}{g_{1}} \mathrm{e}^{-\Delta E / k T}\right\},
$$

where $g_{i}$ is the degeneracy of the ground states $i$. Equation (4) fits well the observed temperature dependence of the line strength (full lines in the right part of Fig. 3) yielding a ground state splitting of $15 \pm 3 \mu \mathrm{eV}$. Similar investigations give $8 \pm 2 \mu \mathrm{eV}$ for InP:Fe. The ground state splittings are in excellent agreement with EPR data for the ${ }^{6} A_{1}(S)$ ground state of $\mathrm{Fe}^{3+}$ [13] which splits into a doublet by second order spin-spin and spin-orbit interaction. Thus, temperature dependent CAS experiments unambiguously prove $\mathrm{Fe}^{3+}$ as a ground state of the observed transitions. All fine structure lines $a^{\prime}-e^{\prime}$ and $a, b$ have a different temperature 

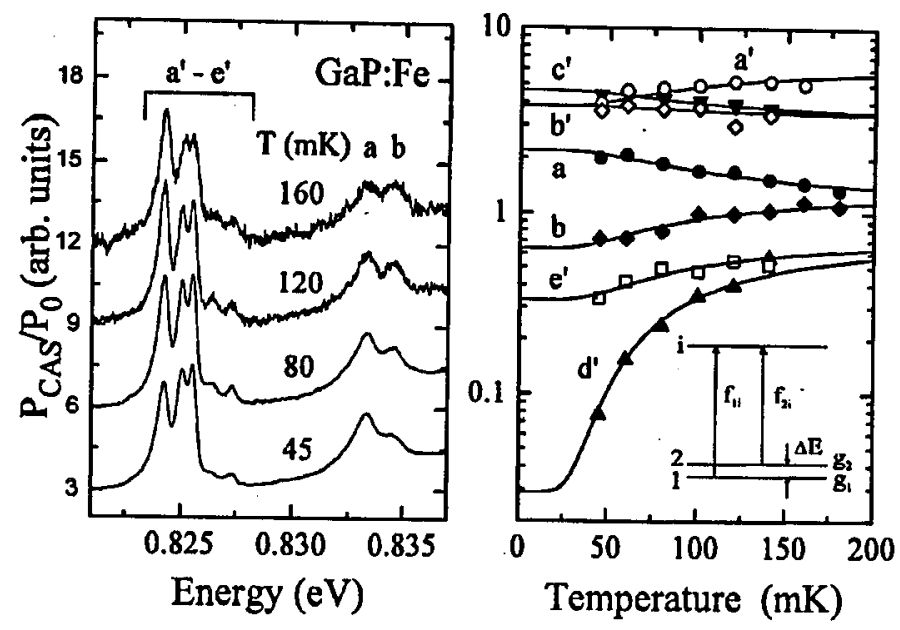

Fig. 3. Temperature dependent CAS spectra of semi-insulating GaP:Fe. The right part shows high-resolution spectra of the fine structure lines attributed to the formation of the $\left(\mathrm{Fe}^{2+}, h\right)$ complex and the left part shows the development of the line intensities with temperatures. Full lines represent a fit with Eq. (4).

dependence. Obviously, all lines have different excited states excluding any interpretation of lines $a, b$ as phonon replica. Especially, lines $a^{\prime}$ and $a$ as well as $b^{\prime}$ and $b$ behave totally different showing the different character of the respective excited states. In addition, the lines in InP show qualitatively the same behavior [12] calling for a general interpretation.

The excited states of the fine structure transitions are related to the $\mathrm{Fe}^{3+/ 2+}$ charge transfer transition. $\mathrm{Fe}^{2+}$ is negatively charged with respect to the host lattice and binds the generated hole via Coulomb interaction. The resulting $\left(\mathrm{Fe}^{2+}, \mathrm{h}\right)$ complex resembles a shallow acceptor though it differs in many aspects. The $\left(\mathrm{Fe}^{2+}, \mathrm{h}\right)$ complex is an excited state of the $\mathrm{Fe}^{3+}$ center and recombines nonradiatively. No corresponding luminescence has been observed yet. In principle the electronic structure of the $\left(\mathrm{Fe}^{2+}, \mathrm{h}\right)$ complex depends on the structure of $\mathrm{Fe}^{2+}$ and the acceptor-like hole states as indeed observed in the spectra. Two additional effects have to be taken into account. First, hybridization of $\mathrm{Fe} 3 d$ states with valence band states leads to a smeared out charge distribution reducing the binding energy of the shallow hole [14]. Second, both the four holes strongly localized in the $\mathrm{Fe} 3 d$ shell and the shallow hole have an angular momentum leading to a term interaction which leads to new combined eigenstates.

The quintet $a^{\prime}-e^{\prime}$ is attributed to the $\left(\mathrm{Fe}^{2+}\left({ }^{5} E\right), \mathrm{h}_{1 S}\right)$ complex with the shallow hole in its $1 S_{3 / 2}$ ground state (Fig. 4). The observed line splittings are reduced by about $50 \%$ compared to those of isolated $\mathrm{Fe}^{2+}$. An exchange interaction of the same order of magnitude as the spin-orbit interaction might change the energy and character of the states. The lines $a$ and $b$ are attributed to the $\left(\mathrm{Fe}^{2+}\left({ }^{5} E\right), \mathrm{h}_{2 P}\right)$ complex with the hole in the first excited $2 P_{3 / 2}$ state, which has a much lower probability in the $\mathrm{Fe} 3 d$ shell than the $1 S_{3 / 2}$ state. Thus, both the Coulomb smearing 
and the exchange interaction can be neglected and the fine structure should mirror the $\mathrm{Fe}^{2+}$ term scheme as indeed observed for lines $a, b$ and $A, B, C$ [5]. Additionally, taking the effective mass energy of the $2 P_{3 / 2}$ state [15] and the energy of line $a$ yields very precise values for the position of the $\mathrm{Fe}^{3+/ 2+}$ acceptor level and the binding energy of the $\left(\mathrm{Fe}^{2+}, \mathrm{h}\right)$ complex. The results are summarized in Table and depicted in Fig. 4.

Effective mass type states of transition metal centers are general phenomena in II-VI and III-V semiconductors and should play a major role as intermediate states in carrier capture processes.

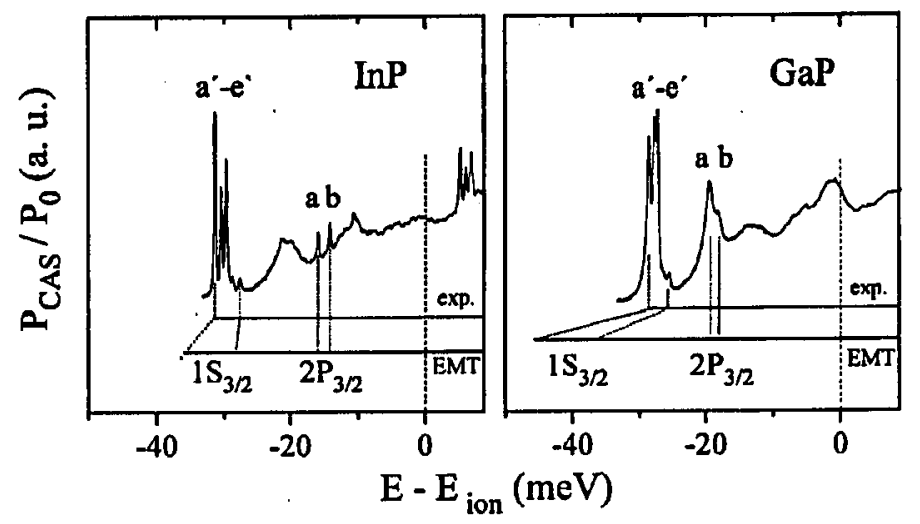

Fig. 4. Assignment of the fine structure lines of the $\left(\mathrm{Fe}^{2+}, h\right)$ complex to different states of the shallow hole according to eflective mass theory (EMT) [15]. The energy is given with respect to the position of the deep $\mathrm{Fe}^{3+/ 2+}$ acceptor.

TABLE

Low temperature parameters of the Fe center in III-V semiconductors. Position of the deep $\mathrm{Fe}^{3+/ 2+}$ acceptor level $\left(E^{3+/ 2+}\right)$, binding energy of the $\left(\mathrm{Fe}^{2+}, h\right)$ complex $\left(E^{\text {shallow }}\right)$, quantum yield $(\eta)$, luminescence lifetime $(\tau)$, and the resulting radiative $\left(W_{\mathrm{r}}\right)$ as well as nonradiative $\left(W_{\mathrm{nr}}\right)$ transition rate of the $\mathrm{Fe}^{2+}\left({ }^{5} T_{2}-{ }^{5} E\right)$ transition. $p$ gives the number of LO phonons needed to span the transition energy.

\begin{tabular}{l|c|c|c|c|c|r|r}
\hline \hline & $\begin{array}{c}E^{3+/ 2+} \\
{[\mathrm{meV}]}\end{array}$ & $\begin{array}{c}E^{\text {shallow }} \\
{[\mathrm{meV}]}\end{array}$ & $\begin{array}{c}\eta \\
\%\end{array}$ & $\begin{array}{c}\tau \\
\mu \mathrm{s}\end{array}$ & $\begin{array}{c}W_{\mathrm{r}} \\
\mathrm{kHz}\end{array}$ & $\begin{array}{r}W_{\mathrm{nr}} \\
\mathrm{kHz}\end{array}$ & $p$ \\
\hline $\mathrm{InP}$ & 800.6 & 31.3 & 47 & $11[16]$ & 43 & 57 & 9.6 \\
$\mathrm{GaP}$ & 851.8 & 28.1 & 26 & $6.6[17]$ & 39 & 114 & 10.3 \\
$\mathrm{GaAs}$ & 510.8 & 22.5 & 35 & $8.5[18]$ & 41 & 77 & 11.1 \\
$\mathrm{ZnS}[25]$ & & & 10 & 5.5 & 18 & 165 & 9.9
\end{tabular}




\section{Radiative and nonradiative transition rates of iron}

Radiative $\left(W_{\mathrm{r}}\right)$ and nonradiative $\left(W_{\mathrm{nr}}\right)$ relaxation rates are normally not directly accessible. Experiments yield the luminescence lifetime $\tau$ and the quantum yield $\eta$ of the radiative transition, both needed to determine $W_{\mathbf{r}}$ and $W_{\mathrm{nr}}$

$$
W_{\mathrm{r}}=\frac{\eta}{\tau} \quad \text { and } \quad W_{\mathrm{nr}}=\frac{1-\eta}{\tau} .
$$

The intracenter ${ }^{5} E-{ }^{5} T_{2}$ transition of $\mathrm{Fe}^{2+}$ leads to structured absorption and emission bands in the near infrared spectral region [9]. Time-resolved luminescence measurements yield lifetimes in the $10 \mu \mathrm{s}$ range for the ${ }^{5} T_{2}$ excited state at liquid He temperatures [16-18]. The lack of experimental values for the quantum yield $\eta$, however, hampered the discussion of nonradiative relaxation processes yet.

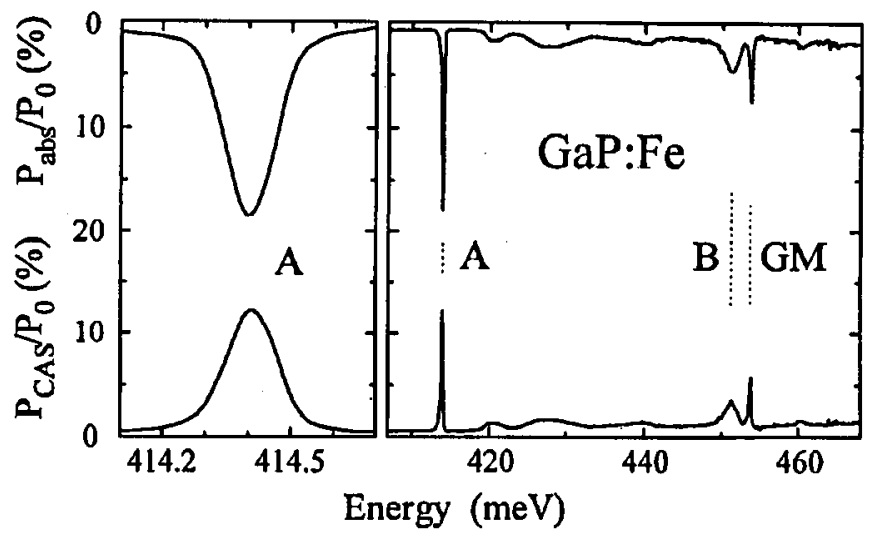

Fig. 5. CAS spectra in comparison to the absorbed light power for the $\mathrm{Fe}^{2+}\left({ }^{5} E-{ }^{5} T_{2}\right)$ transition in semi-insulating GaP:Fe. The powers are given with respect to the incident light power $P_{0}$.

Figure 5 gives simultaneously recorded absorption and CAS spectra for semi-insulating GaP:Fe. Both spectra have almost the same shape resembling the typical low temperature ${ }^{5} E-{ }^{5} T_{2}$ absorption. Lines $A$ and $B$ are dipole-allowed $\Gamma_{1}-\Gamma_{5}$ transitions and the resonance $G M$ is attributed to a local mode replica of line $A$. Comparing the magnitudes of $P_{\mathrm{CAS}}$ and $P_{\mathrm{abs}}$ it is obvious that relaxation from the excited ${ }^{5} T_{2}$ level generates a considerable number of phonons in the sample. Using Eq. (3) an external quantum efficiency $Q$ of $(26 \pm 4) \%$ is determined for $\mathrm{GaP}$. Similar experiments for InP and $\mathrm{GaAs}$ yield $(47 \pm 5) \%$ and $(35 \pm 5) \%$, respectively.

In general the external quantum efficiency $Q$ derived from CAS experiments differs from the quantum yield $\eta$ of a transition, since relaxation via intermediate states, phonon-assisted emission and reabsorption of luminescence influence $P_{\mathrm{CAS}}$. For the ${ }^{5} T_{2}-{ }^{5} E$ transition of $\mathrm{Fe}^{2+}$, however, no intermediate states exist, the phonon sideband in luminescence is weak and reabsorption can be excluded from a comparison of high quality samples of different shape and Fe content. Thus, 
in the case of the internal $\mathrm{Fe}^{2+}$ transition $Q$ is a good approximation for $\mathrm{h}$ in view of the experimental error.

Table compiles the experimental results for the quantum yields $\eta$ and the lifetimes $\tau$ as well as the calculated (Eq. (5)) radiative $W_{\mathrm{r}}$ and nonradiative $W_{\mathrm{nr}}$ relaxation rates for the $\mathrm{Fe}^{2+}\left({ }^{5} T_{2}-{ }^{5} E\right)$ transition. The variation of $\tau$ and $\eta$ results from the variation of $W_{\mathrm{nr}}$ whereas $W_{\mathrm{r}}$ is within the margins of error equal in the III-V semiconductors.

Usually nonradiative relaxation of deep centers takes place by multiphonon emission involving optical phonons, which is described via a configuration coordinate model [19]. The nonradiative relaxation rate depends on the number of phonons $p$ needed to span the transition energy and the electron-phonon coupling strength quantified by the Huang-Rhys factor $S$. In the weak coupling limit $(S \approx 0) W_{\mathrm{nr}}$ decreases exponentially with increasing $p$. For $\mathrm{Fe}^{2+}$ no such trend is observed (Table) and, additionally, $W_{\mathrm{nr}}$ is too high for the weak coupling limit. Obviously, the electron-phonon interaction determines $W_{\mathrm{nr}}$. Fine structure calculations give information on the electron-phonon coupling. The ${ }^{5} E$ ground state experiences a weak coupling to $E$-type modes only [20], whereas for the excited ${ }^{5} T_{2}$ state a moderate dynamical Jahn-Teller coupling has been proposed [21]. The calculations show that the electron-phonon interaction in the ${ }^{5} E$ ground state can be neglected in view of nonradiative relaxation but they give no reliable numbers to discuss the observed variation of $W_{\mathrm{nr}}$.

The Jahn-Teller coupling in the ${ }^{5} T_{2}$ state can be evaluated on the basis of isotope shifts observed for the zero phonon absorption lines [21, 22]. The mass dependence of impurity states is a consequence of mass sensitive local phonons $\left(T_{2}\right.$ modes) contributing to the total energy and the electron-phonon coupling [23]. Isotope shifts of optical transitions are a difference effect depending on both the

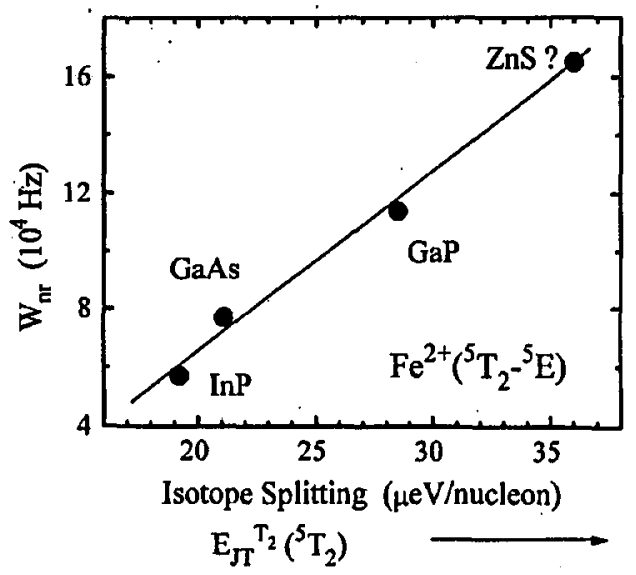

Fig. 6. The nonradiative relaxation rate $W_{\mathrm{nr}}$ of the $\mathrm{Fe}^{2+}\left({ }^{5} T_{2}-{ }^{5} E\right)$ transition versus the respective isotope splitting. The isotope shift is a measure for the dynamical Jahn-Teller coupling of the excited ${ }^{5} T_{2}$ state to local $T_{2}$ modes. 
excited $E_{\text {exc }}$ and ground $E_{\mathrm{gr}}$ state energy and for intracenter $3 d$ transitions a consequence of the vibronic character of the involved states [24]. For $\mathrm{Fe}^{2+}$ the Jahn-Teller coupling in the ${ }^{5} E$ ground state can be neglected, giving

$$
\frac{\mathrm{d} E_{\text {opt }}}{\mathrm{d} M}=\frac{\mathrm{d} E_{\text {exc }}}{\mathrm{d} M}-\frac{\mathrm{d} E_{\mathrm{gr} .}}{\mathrm{d} M}=\left(\frac{\mathrm{d} E_{\mathrm{s} T_{2}}}{\mathrm{~d} \hbar \omega_{T_{2}}}-\frac{3}{2}\right) \frac{\mathrm{d} \hbar \dot{\omega}_{T_{2}}}{\mathrm{~d} M}>0,
$$

whereby the contribution of the local $T_{2}$ mode to the energy of the ${ }^{5} T_{2}$ state depends on the electron-phonon coupling strength. A detailed discussion of Eq. (6) is given in Refs. [23, 24]. Equation (5) predicts a positive isotope shift in agreement with experiment [21,22] and makes its magnitude a measure for the coupling of the ${ }^{5} T_{2}$ excited state to a local $T_{2}$ mode. Figure 6 shows a clear correlation between the nonradiative transition rate $W_{\mathrm{nr}}$ and the respective isotope shift. This demonstrates the crucial role of the dynamical Jahn-Teller coupling of the excited ${ }^{5} T_{2}$ state to a local $T_{2}$ mode for the multiphonon relaxation process. A dynamical Jahn-Teller effect is found to enhance the nonradiative transition rate by orders of magnitude. The higher nonradiative transition rate observed for $\mathrm{ZnS}$ [25] (Table) corresponds well to the stronger electron-phonon coupling in the II-VI compounds. From $W_{\mathrm{nr}}$ we estimate an isotope splitting of $36 \mu \mathrm{eV} /$ nucleon which is smaller than the inhomogeneous linewidth.

\section{Absorption of quantum dots}

Recent progress of epitaxial growth in the coherent island Stranski-Krastanov mode allows the fabrication of nm-scale QDs, e.g. [26-29]. The formation of high density ordered arrays of pyramidal InAs QDs on GaAs can be achieved under particular growth conditions [30,31]. Such QD ensembles show a broad excitonic luminescence peak with a linewidth of about $50 \mathrm{meV}$ due to size fluctuations. The existence and nature of the relaxation processes leading to ground state population after nonresonant excitation is controversially discussed [32].

Figure 7 compares photoluminescence (PL), CAS and PL excitation (PLE) spectra of an InAs/GaAs QD structure grown by conventional solid source molecular beam epitaxy as described in Ref. [30]. A single sheet of self-organized QDs is formed from a $4 \mathrm{ML}$ InAs layer grown at $480^{\circ} \mathrm{C}$ and overgrown with GaAs. TEM images show highly symmetric InAs pyramids with a base-plane size of about $12 \mathrm{~nm}$ and a density of $10^{11} \mathrm{~cm}^{-2}$ [30]. The InAs QDs are located on a 1.7 ML thick InAs wetting layer. Numerical calculations indicate a strong relaxation of the biaxial strain in the coherent InAs QDs and show that only one electron but several hole states are confined in the QD. A detailed description of the calculations is given in Ref. [33].

The PL spectrum is dominated by QD emission peaking at $1.11 \mathrm{eV}$ showing only weak luminescence from the GaAs barrier (Fig. 7). The calculations yield a QD ground state transition energy of $1.11 \mathrm{eV}$ for $12 \mathrm{~nm}$ pyramids in good agreement with TEM results. The CAS spectrum reveals 3 absorption lines related to the QD structure. The low energy peak around $1.11 \mathrm{eV}$ coincides with the QD luminescence. For isolated QDs having a $\delta$-function density of states absorption and emission transitions have to coincide in energy, except for the case that defect states are involved. Thus, the CAS spectrum proves coherent InAs QDs to 


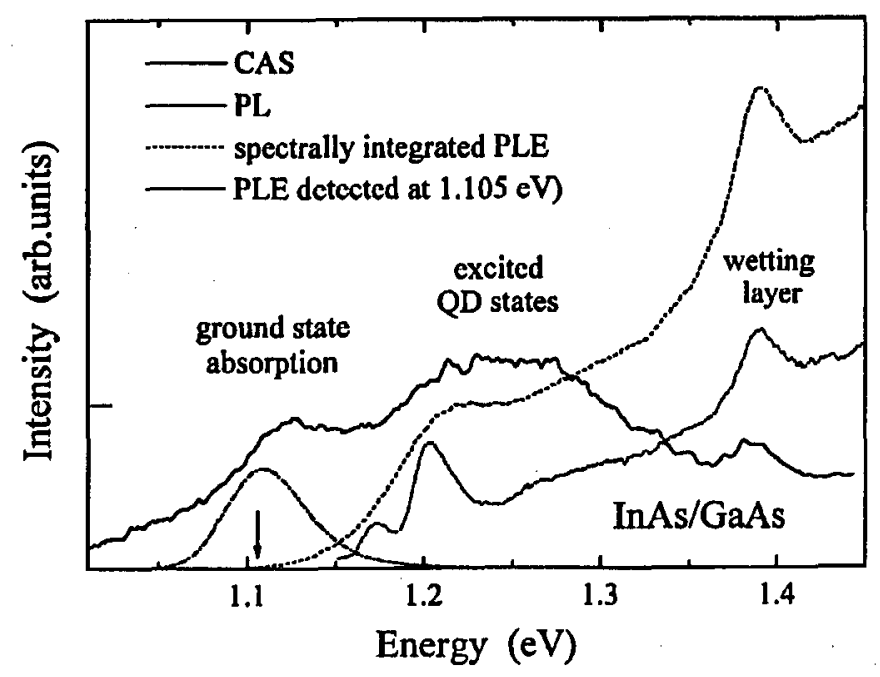

Fig. 7. Luminescence (excited at $1.96 \mathrm{eV}$ ), CAS and PLE spectra of small InAs/GaAs QDs. In addition to the PLE spectrum detected at $1.105 \mathrm{eV}$ the spectrally integrated PLE (as described in the text) is shown.

be defect-free. The second broad absorption between 1.2 and $1.3 \mathrm{eV}$ can be explained with parity-allowed transitions involving excited hole states. The third absorption at $1.388 \mathrm{eV}$ results from the InAs wetting layer. PLE detecting the excitation of photon emission represents an experimental technique complementary to CAS. The PLE spectrum detected at the maximum of the QD luminescence differs considerably from the CAS spectrum (Fig. 7). Both spectra show the wetting layer absorption, but instead of broad absorption into excited hole states PLE shows two sharp lines. CAS spectra reflect the inhomogeneous broadening of the QD ensemble, whereas PLE is independent of this inhomogeneity but is not able to reveal resonant absorption. The inhomogeneous broadening is obtained from a summation over PLE spectra recorded at different positions of the QD luminescence, whereby the resonant part dominated by stray light has been artificially suppressed. The spectrally integrated PLE spectrum corresponds well to the CAS spectrum (Fig. 7).

The energy shift of the PLE lines with respect to the detection energy corresponds to multiples of the InAs QD LO phonon energy of about $32 \mathrm{meV}$ independent of the detection energy proving multiphonon processes to be important for carrier relaxation in these QDs. The agreement between the CAS and the spectrally integrated PLE spectrum shows that parity-allowed transitions into excited hole states are responsible for the observed intensity distribution.

\section{Conclusion}

The basic principles of calorimetric absorption spectroscopy at temperatures between $2 \mathrm{~K}$ and $45 \mathrm{mK}$ were discussed. CAS is a highly sensitive absorption 
method allowing to quantify nonradiative relaxation processes. Even, using low power light sources $\alpha d$ products of less than $10^{-5}$ can be detected.

Examples presented here include the detection of small absorption signals of a single InAs/GaAs QD layer, the analysis of weak fine structure at the low energy onset of the $\mathrm{Fe}^{3+/ 2+}$ charge transfer absorption bands in semi-insulating III-V semiconductors and the evaluation of nonradiative relaxation processes of the $\mathrm{Fe}^{2+}$ center.

\section{Acknowledgment}

Parts of this work were supported by DFG in the framework of $\mathrm{Sfb} 296$ and by Volkswagen foundation.

\section{References}

[1] W.B. Jackson, N.M. Amer, A.C. Boccara, D. Fournier, Appl. Optics 20, 1333 (1981).

[2] D. Bimberg, A. Bubenzer, Appl. Phys. Lett. 38, 803 (1981).

[3] D. Bimberg, T. Wolf, J. Böhrer, in: Advances in Nonradiative Processes in Solids, Ed. B. DiBartolo, Plenum Press, New York 1991, p. 577.

[4] L. Podlowski, A. Hoffmann, I. Broser, J. Cryst. Growth 117, 698 (1992).

[5] A. Juhl, A. Hoffmann, D. Bimberg, H.-J. Schulz, Appl. Phys. Lett. 50, 1292 (1987).

[6] I. Broser, L. Podlowski, P. Thurian, R. Heitz, A. Hoffmann, J. Cryst. Growth 138, 809 (1994).

[7] A. Juhl, D. Oertel, R. Bauer, C. Maczey, D. Bimberg, M. Razeghi, Acta Phys. Pol. A 69, 877 (1986).

[8] D. Bimberg, J. Böhrer, A. Krost, J. Vac. Sci. Technol. A 12, 1039 (1994).

[9] B. Clerjaud, J. Phys. C, Solid Slate Phys. 18, 3615 (1985).

[10] K. Thonke, K. Pressel, Phys. Rev B 44, 13418 (1991).

[11] T. Wolf, D. Bimberg, W. Ulrici, Phys. Rev B 43, 10004 (1991).

[12] L. Podlowski, R. Heitz, T. Wolf, A. Hoffmann, D. Bimberg, I. Broser, W. Ulrici, Mater. Sci. Forum 143-147, 311 (1994).

[13] H.H. Woodbury, G.W. Ludwig, Bull. Am. Phys. Soc. 6, 118 (1961).

[14] V.N. Fleurov, K.A. Kikoin, Solid State Commun. 58, 157 (1986).

[15] A. Baldereschi, N. Lipari, Phys. Rev. B 9, 1525 (1974).

[16] P.B. Klein, J.E. Fournaux, R.L. Henry, Phys. Rev. B 29, 1947 (1984).

[17] P.B. Klein, K. Weiser, Solid State Commun. 41, 365 (1982).

[18] K. Pressel, G. Bohnert, G. Rückert, A. Dörnen, K. Thonke, J. Appl. Phys. 71, 5703 (1992).

[19] P.T. Landsberg, Recombination in Semiconductors, Cambridge University Press, Cambridge 1993.

[20] E.E. Vogel, O. Mualin, M.A. Rivera, J. Rivera-Iratchet, Phys. Rev. B 44, 729 (1991).

[21] K. Pressel, G. Rückert, A. Dörnen, K. Thonke, Phys. Rev. B 46, 13171 (1992).

[22] G. Rueckert, K. Pressel, A. Dörnen, K. Thonke, W. Ulrici, Phys. Rev. B 46, 13207 (1992).

[23] A. Hoflmann, U. Scherz, J. Cryst. Growth 101, 385 (1990). 
[24] R. Heitz, A. Hoftmann, I. Broser, Phys. Rev. B 45, 8977 (1992).

[25] L. Podlowski, R. Heitz, P. Thurian, A. Hoffmann, I. Broser, J. Lumin. 58, 252 (1994).

[26] D. Leonard, M. Krishnamurthy, C.M. Reaves, S.P. DenBaars, P.M. Petroff, Appl. Phys. Lett. 63, 3203 (1993).

[27] J.M. Moison, F. Houzay, F. Barthe, L. Leprince, E. Andro, O. Vatel, Appl. Phys. Lett. 64, 196 (1994).

[28] M. Grundmann, J. Christen, N.N. Ledentsov, J. Böhrer, D. Bimberg, S.S. Ruvimov, P. Werner, U. Richter, U. Gösele, J. Heydenreich, V.M. Ustinov, A.Yu. Egorov, A.E. Zhukov, P.S. Kop'ev, Zh.I. Alferov, Phys. Rev. Lett. 20, 4043 (1995).

[29] N.N. Ledentsov, M. Grundmann, N. Kirstaedter, J. Christen, R. Heitz, J. Böhrer, F. Heinrichsdorff, D. Bimberg, S.S. Ruvimov, P. Werner, U. Richter, U. Gösele, J. Heydenreich, V.M. Ustinov, A.Yu. Egorrov, M.V. Maximov, P.S. Kop'ev, Zh.I. Alferov, in: Proc. 22nd Int. Conf. Phys. Semicond., Ed. D. Lockwood, World Scientific, Singapore 1995, p. 1855.

[30] M. Grundmann, N.N. Ledentsov, R. Heitz, L. Eckey, J. Christen, J. Böhrer, D. Bimberg, S.S. Ruvimov, P. Werner, U. Richter, J. Heydenreich, V.M. Ustinov, A.Yu. Egorov, A.E. Zhukov, P.S. Kop'ev, Zh.I. Alferov, Phys. Status Solidi B 188, 249 (1995).

[31] S.S. Ruvimov, P. Werner, K. Scheerschmidt, J. Heydenreich, U. Richter, N.N. Ledentsov, M. Grundmann, D. Bimberg, V.M. Ustinov, A.Yu. Egorov, P.S. Kop'ev, Zh.I. Alferov, Phys. Rev. B 51, 14766 (1995).

[32] H. Benisty, C.M. Sotomayor Torres, C. Weisbuch, Phys. Rev. B 44, 10945 (1991).

[33] M. Grundmann, O. Stier, D. Bimberg, to be published in Phys. Rev. B. 